\title{
Cardiomyocyte transcription is controlled by combined mineralocorticoid receptor and circadian clock signalling
}

\author{
Elizabeth K Fletcher1,2,3,*, Monica Kanki1,*, James Morgan¹, David W Ray4,5, Lea M Delbridge², Peter J Fuller¹, \\ Colin D Clyne1 and Morag J Young1
}

${ }^{1}$ Centre for Endocrinology and Metabolism, Hudson Institute of Medical Research, Clayton, Victoria, Australia 2Department of Physiology, The University of Melbourne, Parkville, Victoria, Australia

3Tufts Medical Center, Boston, Massachusetts, USA

${ }_{4}^{4}$ NIHR Oxford Biomedical Research Centre, John Radcliffe Hospital, Oxford, UK

${ }^{5}$ Oxford Centre for Diabetes, Endocrinology and Metabolism, University of Oxford, Oxford, UK

Correspondence should be addressed to M J Young: morag.young@hudson.org.au

*(E K Fletcher and M Kanki contributed equally to this work)

\begin{abstract}
We previously identified a critical pathogenic role for mineralocorticoid receptor (MR) activation in cardiomyocytes that included a potential interaction between the MR and the molecular circadian clock. While glucocorticoid regulation of the circadian clock is undisputed, studies on MR interactions with circadian clock signalling are limited. We hypothesised that the MR influences cardiac circadian clock signalling, and vice versa. Aldosterone or corticosterone (10 nM) regulated Cry1, Per1, Per2 and ReverbA (Nr1d1) gene expression patterns in H9c2 cells over $24 \mathrm{~h}$. MR-dependent regulation of circadian gene promoters containing GREs and E-box sequences was established for CLOCK, Bmal, CRY1 and CRY2, PER1 and PER2 and transcriptional activators CLOCK and Bmal modulated MR-dependent transcription of a subset of these promoters. We also demonstrated differential regulation of MR target gene expression in hearts of mice $4 \mathrm{~h}$ after administration of aldosterone at $08: 00 \mathrm{~h}$ vs 20:00 h. Our data support MR regulation of a subset of circadian genes, with endogenous circadian transcription factors CLOCK and BMAL modulating the response. This unsuspected relationship links MR in the heart to circadian rhythmicity at the molecular level and has important implications for the biology of MR signalling in response to aldosterone as well as cortisol. These data are consistent with MR signalling in the brain where, like the heart, it preferentially responds to cortisol. Given the undisputed requirement for diurnal cortisol release in the entrainment of peripheral clocks, the present study highlights the MR as an important mechanism for transducing the circadian actions of cortisol in addition to glucocorticoid receptor (GR) in the heart.
\end{abstract}

\section{Key Words}

- mineralocorticoid receptor

- circadian clock

- Bmal

- CLOCK

- Per2

- decorin 


\section{Introduction}

The mineralocorticoid receptor (MR) is a ligand-activated transcription factor best known for regulating sodium/ potassium and water in the kidney in response to aldosterone (Fuller \& Young 2005). The MR has equivalent high affinity of $\mathrm{Kd} \sim 0.5-1.0 \mathrm{nM}$ for aldosterone and cortisol (corticosterone in rodents); the $\mathrm{Kd}$ for cortisol-binding glucocorticoid receptor (GR) is lower $\sim 10-25 \mathrm{nM}$. Given that glucocorticoids circulate at levels 100-1000-fold higher than that of aldosterone, it is the co-localisation of 11ß-hydroxysteroid dehydrogenase type 2 (HSD2) in the kidney and other epithelial tissues that allows aldosterone to bind and activate the MR. In most non-epithelial tissues the MR can respond to both aldosterone and glucocorticoids due to the absence of HSD2, but are normally occupied by cortisol. In cell types expressing HSD2 evidence suggests that MR activation by cortisol (when HSD2 is inhibited) is similar to aldosterone. Physiological levels of aldosterone can regulate cardiomyocytes, although the mechanisms remain unclear, and cortisol opposes aldosterone actions. However, in cardiomyocytes, inappropriate MR activation by mineralocorticoids or glucocorticoids promotes inflammation and fibrosis and remodelling of electrical signalling to impact myocardial function in chronic disease as well as myocardial recovery following acute cardiac events (Bienvenu et al. 2015). To understand how MR regulates cardiomyocyte function, genome-wide analysis of transcriptional responses in hearts from wild-type mice vs mice lacking the MR selectively in cardiomyocytes (myoMRKO) identified several circadian clock genes that were differentially regulated in naïve cardiomyocyte MR-null mice: PERIOD 2 (PER2), Circadian Locomotor Output Cycles Kaput (CLOCK), ReverbA (Nr1d1), neuronal PAS domain-containing protein 2 (Npas2) and Ep300 (Rickard et al. 2012). We recently identified a number of novel MR targets in the heart including an interaction between the MR and the cardiac circadian clock where MR-mediated cardiac inflammation and fibrosis is blunted in animals harbouring a mutant CLOCK protein that renders the molecular circadian clock non-oscillatory (Fletcher et al. 2017).

The circadian clock is a ubiquitously expressed, tightly controlled set of transcriptional regulators; the 'central' clock in the suprachiasmatic nucleus (SCN) perceives time of day and entrains 'tissue' clocks via hypothalamic control of cortisol/corticosterone release to enable peripheral tissue to anticipate environmental stimuli (Takahashi \& Zatz 1982). The GR and MR are not expressed in the SCN but the GR serves as a zeitgeber of the circadian clock in peripheral tissues (Kino \& Chrousos 2011a). Circadian rhythm is driven by the oscillation of the molecular clock, which comprises BMAL (brain and muscle aryl hydrocarbon receptor nuclear translocator (ARNT)-like) and CLOCK heterodimers transcriptionally regulating PER1, 2 and 3 and CRYPTOCHROME (CRY1 and 2) genes. BMAL-CLOCK proteins are in turn inhibited by PER/CRY to establish the periodicity of the system. Other circadian genes - that is Npas2, casein kinase $1 \varepsilon(\mathrm{CsnK} 1 \varepsilon)$ and ReverbA - reinforce 'time keeping' of the clock (Caratti et al. 2018). Many circadian clock proteins are transcription factors and directly regulate the rhythmic expression of thousands of genes via E-box response elements in target gene promoters (Durgan \& Young 2010).

Recent epidemiological and experimental studies have unambiguously linked circadian misalignment to cardiovascular disease, although the underlying mechanisms are unknown. While GR regulation of the peripheral molecular clock is well-accepted (Yamamoto et al. 2005, Reddy et al. 2007), only a limited number of studies suggest direct MR interaction with the cardiac molecular clock (Tanaka et al. 2007, Stow et al. 2012). Aldosterone induces expression of PER1 and PER2 in cardiomyocytes and renal cells, although experimental levels of ligand $(1 \mu \mathrm{M})$ were used in one study (Stow et al. 2012). Although glucocorticoids are released in a circadian manner and have an undisputed role in the entrainment of peripheral clocks to the central pace maker in the SCN, GR signalling does not account for all cortisol effects on the peripheral clock (Tanaka et al. 2007). Serum aldosterone also shows circadian variation; albeit at levels substantially lower than for glucocorticoids (Lamarre-Cliche et al. 2005). Given that the MR also responds to glucocorticoids in the heart it is likely that the MR regulates a subset of circadian clock genes in response to oscillating glucocorticoid levels across the day.

Core circadian clock proteins, Bmal and CLOCK, are also transcription factors and regulate target genes via binding to 'E-box' response elements. Previous studies have identified two forms of E-box: canonical (inverted palindrome) and non-canonical (non-palindromic sequence) with similar transcriptional activity (Singletary et al. 2008, Yoshitane et al. 2009). To date studies investigating GRE-dependent interactions with molecular clock pathways have largely focussed on GR-dependent signalling. However it is well known that the MR regulates transcription via most, but not all, GRE sequences and that overlap may occur between MR and GR signalling at some response GREs (Le Billan et al. 2015, Mahfouz et al. 2016).

In the present study we sought to determine if these transcriptional regulatory sites can act together to control 
MR-dependent circadian and MR target genes expression in cardiomyocytes. Our goal therefore was to define molecular signalling mechanisms for MR/circadian clock and their combined regulation of transcriptional responses in cardiomyocytes that may have therapeutic utility. Our data provide novel insights for direct regulation of target genes by MR, Bmal and/or CLOCK signalling in cardiomyocytes and time-of-day-dependent MR signalling outcomes were demonstrated in vivo in mouse hearts.

\section{Methods}

\section{Cell culture}

H9c2 rat cardiomyocytes, mouse vascular smooth muscle cells (MoVas) and human embryonic kidney cells (HEK293) were obtained from ATCC and maintained in Dulbecco's Modified Eagle's Medium (DMEM; Gibco) supplemented with $10 \%$ foetal calf serum (FCS; Gibco) and $1 \%$ penicillinstreptomycin (Anti-Anti 100x; Gibco) at $37^{\circ} \mathrm{C}$ in $5 \% \mathrm{CO}_{2}$ in a humidified incubator. All cell lines were used at $\leq 20$ passages. For experiment 24 well plates were seeded with 40,000 cells/well. The following day cells were synchronised by serum shock using 50\% FCS in DMEM for $2 \mathrm{~h}$ (Balsalobre et al. 1998). After $2 \mathrm{~h}$ the media was replaced with $5 \%$ charcoal stripped FCS containing 10nM aldosterone (to fully activate the MR) or $10 \mathrm{nM}$ cortisol (to activate the MR but not the GR) $\pm 10 \mu \mathrm{M}$ spironolactone and RNA isolated from sets of cells harvested every $3 \mathrm{~h}$ thereafter.

\section{RNA isolation, CDNA synthesis and RT PCR}

RNA was isolated using the TriRagent (Sigma Aldrich) and DNAse treatment (Applied Biosystems, Life Technologies) as previously described and $250 \mathrm{ng}$ of total RNA reversetranscribed using the Ambion cDNA kit (Life Technologies). Target gene expression was quantified using the Applied Biosystems 7900HT Fast Real-Time PCR system and genespecific primers (Supplementary Table 1, see section on supplementary data given at the end of this article, Sigma Aldrich). Fold change vs vehicle for each treatment and time point was determined using the $\Delta \Delta \mathrm{CT}$ method and using the housekeeping gene hypoxanthine phosphoribosyl transferase to correct for total RNA input in the qRT-PCR.

\section{Identification of transcriptional regulatory sequences in target gene promoters}

The promoter sequences of circadian genes were defined as the $10 \mathrm{~kb}$ region upstream of the transcription start site.
Gene promoters for CLOCK, Bmal, CRY1, CRY2, novel MR target gene decorin (Rickard et al. 2012), Deleted in oesophageal cancer 1 (DEC-1), D site of albumin promoter (albumin D-box) binding protein (DBP), PER1, PER2, ReverbA, NR1F1 (RORA), Thyrotroph Embryonic Factor (TEF) and TIMELESS were analysed. Sequences were identified in Ensembl (http://ensembl.org/) using rat, mouse and human genomes. The $10 \mathrm{~kb}$ promoter sequences were exported in FASTA and UCSC website and BLAT (http://genome.ucsc.edu/cgi-bin/hgBlat) was used to validate the correct location on the relevant genome.

Promoter regions were analysed using MatInspector (www.genomatix.de). GRE hits were divided into positive and negative GREs and sorted according to their core similarity. The online search engine JASPAR (http://www.jaspar.genereg.net/) (version 5.0_APLHA) was used for the identification of both canonical and non-canonical GRE sequences in the individual promoter regions. The location of canonical E-boxes (CACGTG) and non-canonical E-boxes (CACGNG, CACGTT, CATGTG and CATGCG) were assessed in sense and antisense sequences. Other transcription factorbinding sites (AP-1 sites, PAR and C/EBP) identified and reviewed for their proximity to GRE and E-box transcription-binding sites.

Identification of regions of homology between the rat, mouse and human genomes was determined using the online search tool ClustalW2 (http://www.ebi. ac.uk/Tools/msa/clustalw2/) from the EMBL-EBI suit. Sequences used for alignment were human, mouse and rat genomes and were uploaded into the Clustal2W search engine in FASTA format: homosapiens, chromosome_GRCh37_4_56288070_5 mus musculus chromosome_GRCm38_5_76206177_7 rattus norvegus, chromosome_Rnor_5.0_14_3423296 (Supplementary Tables 2, 3, 4, 5, 6, 7 and 8). Upstream promoter regions containing high clustering of putative and established GRE sequences and their proximity to E-box sequences were selected for further analysis (Supplementary Tables 9, $10,11,12,13,14,15,16,17$ and 18).

\section{Plasmids}

The following plasmids were used: pGL3basic (Promega), pGL3-enhancer (Promega), PRshMR (a gift from Prof. R Evans), CNK3 (Ziera et al. 2009), pcDNA3.1(+), pcDNA4.0, pBMP3C, pCKPC4, MMTV.luc and Per1.luc (Fletcher et al. 2017). New luciferase reporter plasmids were generated using amplified DNA from the region of interest 
containing selected GREs and subcloned into a luciferase reporter vector (pGL3 basic or enhancer; Promega) using compatible restriction sites (T4 DNA ligase; NEB, Ipswich, MA, USA). The Bmal luciferase reporter was constructed using pGL4 (Promega). All plasmids were verified by sequencing. The promoter regions validated in the present study are summarised in Supplementary Fig. 1.

\section{Analysis of circadian clock and MR regulation of promoter sequences}

Transactivation studies using luciferase reporters of target gene promoter regions were performed as previously described (Yang et al. 2011). Cells were transfected with $200 \mathrm{ng}$ of GRE-luc reporter, 200ng of CLOCK, Bmal1 or CLOCK or Bmal1 expression vector, and $200 \mathrm{ng}$ MR vector and treated or not with aldosterone $(10 \mathrm{nM})$ and/or spironolactone $(10 \mu \mathrm{M})$. pCDNA3.1 (CLOCK) or pCDNA4.0 (Bmal) served as control DNA in the relevant groups.

\section{MR-mediated transcriptional responses in the heart: 08:00 h vs $20: 00 \mathrm{~h}$}

All procedures involving animals were approved by Monash University Animal Ethics and Biosafety Committees. A total of 68 male mice (18-20g) were randomly assigned to receive no treatment, or a subcutaneous injection of aldosterone $(50 \mu \mathrm{g} / \mathrm{kg})$ or vehicle (saline) at either $08: 00 \mathrm{~h}$ or 20:00 h. Heart and liver tissues from mice receiving no treatment were collected at either 08:00 h $(n=10)$ or 20:00 h $(n=10)$. These groups served as controls for time-dependent changes in gene expression.

Mice treated at $08: 00 \mathrm{~h}$ were culled $4 \mathrm{~h}$ later at $12: 00 \mathrm{~h}$ and mice treated at 20:00 h were culled at 00:00h. The heart and liver were rapidly collected and snap frozen at $-80^{\circ} \mathrm{C}$ until processed for RNA. RT PCR was performed as described.

\section{Statistics}

Data were analysed and prepared using GraphPad Prism 6.2 software package (GraphPad Software). One-way ANOVA with Tukey's multiple comparison post hoc test to identify significant differences between the means of each group was performed for all data sets and data are presented as mean \pm S.E. All treatment groups contained between 10 animals. Cell experiments were repeated four times and performed in triplicate.

\section{Results}

MR signalling modulates cardiac circadian clock gene signalling in a ligand-dependent manner

To establish if MR activation modulates the $24 \mathrm{~h}$ pattern of circadian gene transcription we assessed circadian gene expression following $10 \mathrm{nM}$ aldosterone or $10 \mathrm{nM}$ corticosterone in rat cardiac H9c2 cells synchronised by serum shock. All circadian genes showed oscillating mRNA levels over $24 \mathrm{~h}$ consistent with previous demonstration of peripheral circadian function in cardiomyocytes (Fig. 1). Bmal, CLOCK and Timeless were not regulated by the addition of aldosterone or corticosterone (Fig. 1A). CRY1 and PER1 mRNA levels were significantly increased from $6 \mathrm{~h}$ vs serum shock by both aldosterone and corticosterone and remained elevated at 9 and $12 \mathrm{~h}$ (Fig. 1B). PER2 expression was also significantly increased vs serum shock by corticosterone, at $6 \mathrm{~h}$ and $9 \mathrm{~h}$, and was significantly lower at 18 and $24 \mathrm{~h}$ suggesting a phase shift in PER2 expression in response to corticosterone but not aldosterone. In contrast, both aldosterone and corticosterone significantly reduced ReverbA expression at 9 and $12 \mathrm{~h}$; mRNA levels remained low at later time points for corticosterone, but not aldosterone treatment (Fig. 1B). The addition of spironolactone significantly reversed corticosterone induction of CRY1 and PER1 at the $9 \mathrm{~h}$ time point, whereas CRY1 and ReverbA mRNA levels in response to spironolactone plus corticosterone, or spironolactone plus aldosterone, were not different to serum shock alone (Supplementary Fig. 1). Gene expression in response to hormone plus spironolactone may also reflect a phase shift in the pattern of gene expression rather than a marked change in steadystate mRNA levels at any single time point; that is, the oscillatory profile of gene expression may be shifted, rather than the amplitude of response at a single time point. The $24 \mathrm{~h}$ pattern of gene expression for other circadian genes including CRY2 and Timeless was not modulated by aldosterone or corticosterone in cells synchronised by serum shock (Supplementary Fig. 2).

\section{Identification of transcription factor-binding sites in promoter region of circadian and MR target genes}

$10,000 \mathrm{bp} 5^{\prime}$ of the ATG start site for each circadian or MR target gene was identified on Ensembl, and the rat genome was assessed first given that subsequent validation studies were performed in a H9c2 rat cardiomyocyte cell line; sequences for mouse and human were also assessed to establish between species homology and showed 

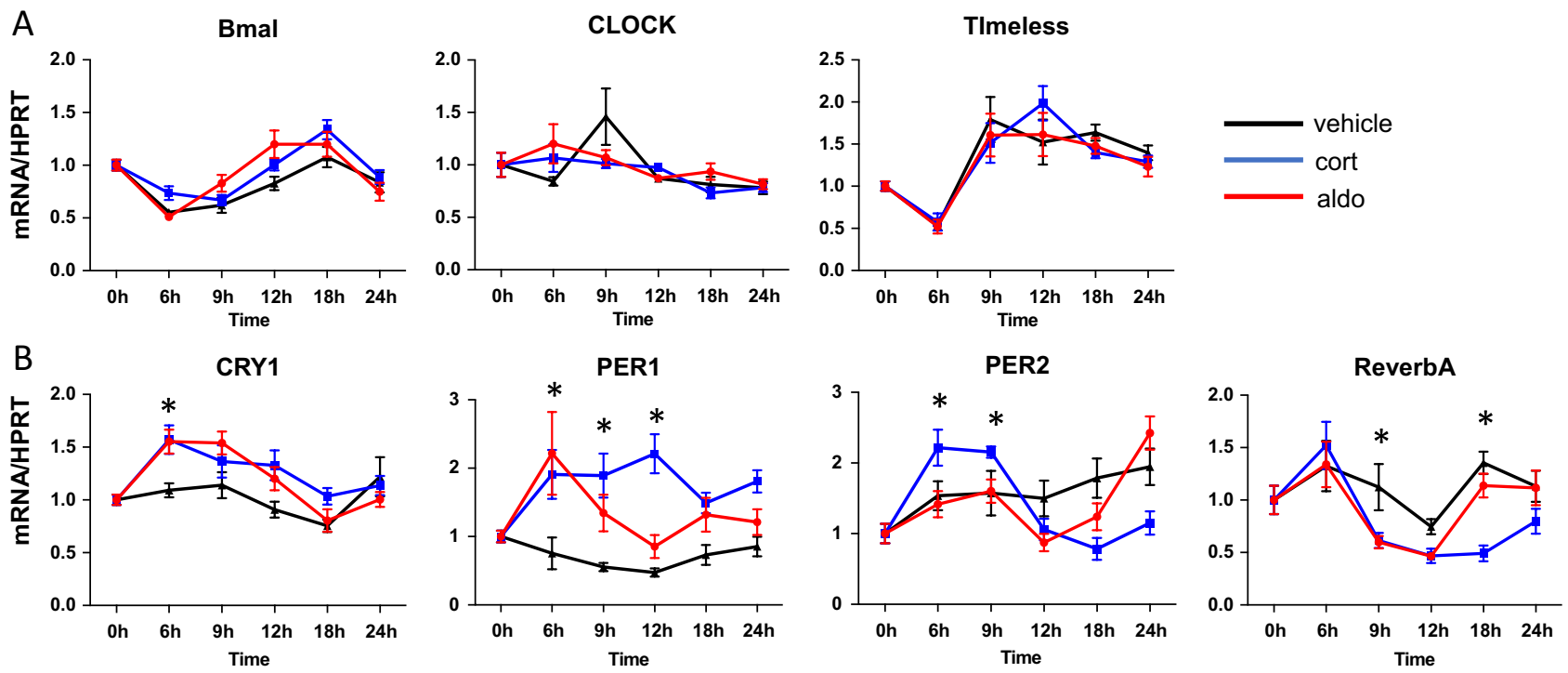

Figure 1

(A) Core circadian genes Bmal1, CLOCK and Timeless are not regulated by $10 \mathrm{nM}$ aldosterone or corticosterone over $24 \mathrm{~h}$ in cardiac $\mathrm{H} 9 \mathrm{c} 2 \mathrm{cells}$. However (B) Cry1, Per1, Per2 and ReverbA are regulated by ligand-activated MR. Data are expressed relative to no treatment at 0 h. Mean \pm s.E.M., $n=6$. $* P<0.05$ vs serum shock alone.

$>50 \%$ homology between human and rodent sequences (Supplementary Table 2). Putative GRE sequences were identified using JASPAR and TransFact online search engines and are summarised in Supplementary Table 3. Circadian transcription factor-binding sites, E-boxes, were also identified and their proximity to GRE sequences noted within the $10 \mathrm{~kb} 5^{\prime}$ region. Our analyses showed the promoters for each circadian gene contained one or more canonical E-box sequences, except CRY2. The promoter region and untranslated $5^{\prime}$ region of CRY2 contained several non-canonical E-box sequences, as did other peripheral molecular clock genes (Supplementary Table 3).
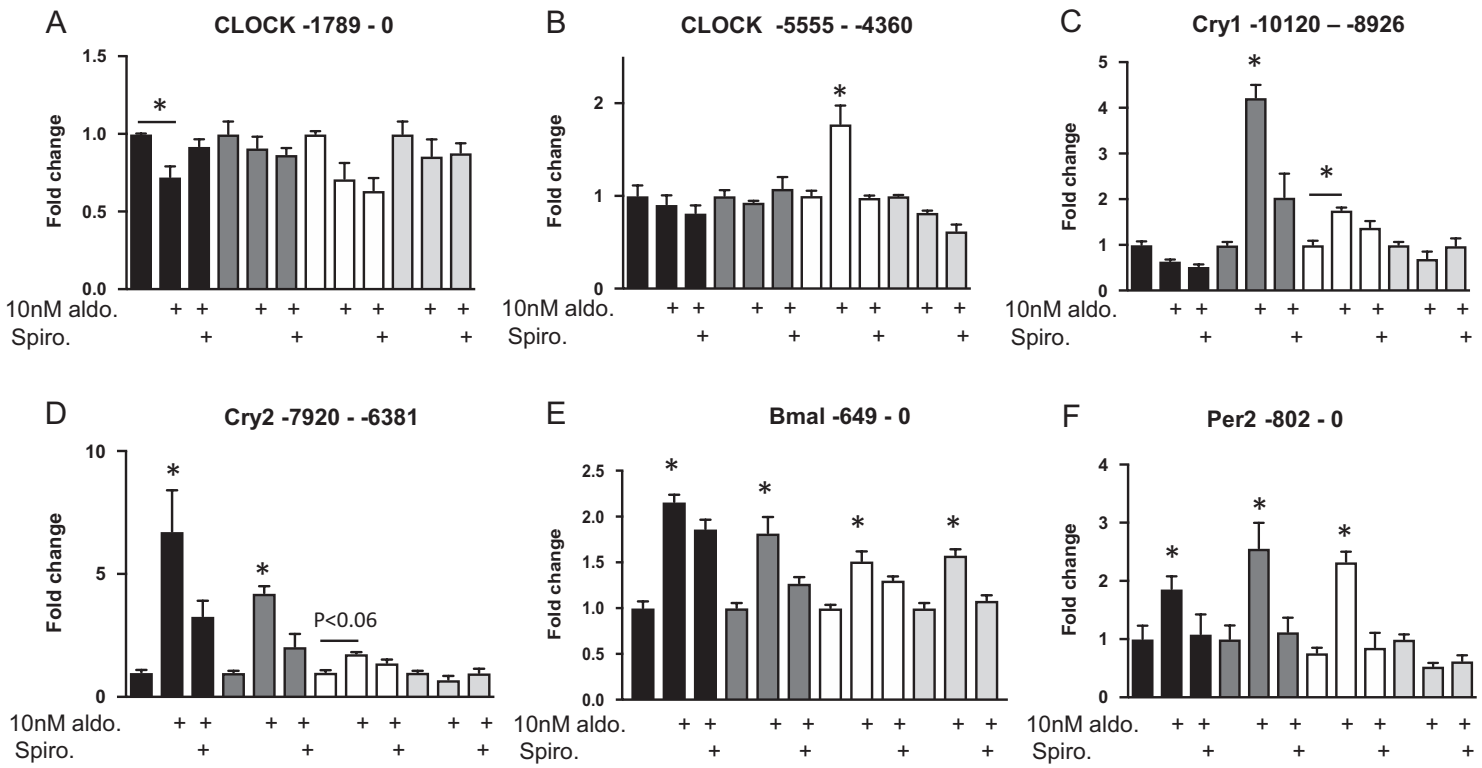

\section{Figure 2}

Regulation of circadian gene promoters by aldosterone is modified by overexpression of Bmal (dark grey bars), Clock (white bars) or Bmal + CLOCK (light grey bars) vs empty vector (black bars). CLOCK and Bmal repress or enhance aldosterone-mediated transactivation of the gene promoters alone ((A) CLOCK, (B) CLOCK, (C) Cry1, (D) Cry2 and (F) Per2) or together ((D) Cry2, (E) Bmal and (F) Per2). Data are presented as mean \pm S.E.M., $n=4$ experiments performed in triplicate, $* P<0.5$ vs vehicle. 
Despite a previous report on a functional overlap between a GRE and a non-canonical E-box in the promoter region of PER2 we did not identify an overlap between GRE and E-box sequences (Cheon et al. 2013). The DEC1 promoter contained a non-canonical E-box and a GRE separated by $2 \mathrm{bp}$ at about $9 \mathrm{~kb}$ upstream. All other predicted E-boxes were located within $80-400$ bps of a GRE sequence. The 5' promoter region for cardiomyocyte MR-regulated gene decorin also contained GREs and E-box sequences (Table 1 and Supplementary Table 3).

\section{Aldosterone regulates select circadian gene promoters and this is modulated by co-expression of CLOCK and Bmal}

Gene promoter regions selected for luciferase validation contained GREs with a high score from JASPAR searches (>10) and were most similar to the consensus GRE (Chandler etal. 1983). Previously published GREs were identified in the selected regions containing novel GREs and E-box elements (Reddy et al. 2012, Cheon et al. 2013) (Supplementary Fig. 3 and Supplementary Table 2). The activity of luciferase reporter constructs containing selected regions of interest was assessed in H9c2 cells. The CLOCK promoter contained two regions of interest: ( -1789 to 0$)$ and ( -5555 to -4360$)$; transactivation by CLOCK -1789 to 0 was modestly but significantly reduced by $10 \mathrm{nM}$ aldosterone, a response that was lost when Bmal, CLOCK or Bmal plus CLOCK were co-transfected with MR. In contrast CLOCK ( -5555 to -4360 ) only responded to aldosterone when CLOCK was overexpressed (Fig. 3A and B). Similarly the $(-10,120$ to -8926$) 5^{\prime}$ region for CRY1 was induced by aldosterone

Table 1 Table of genes selected for further testing listing the total number of GREs and negative GREs identified in their promoters.

\begin{tabular}{|c|c|c|c|}
\hline Gene & GRE & nGRE & $\begin{array}{c}\text { GREs selected for } \\
\text { testing }\end{array}$ \\
\hline CLOCK & 14 & - & 4 \\
\hline Bmal1 & 10 & 1 & 0 \\
\hline CRY1 & 8 & 2 & 5 \\
\hline CRY2 & 5 & 2 & 4 \\
\hline PER1 & 3 & 1 & 2 \\
\hline PER2 & 5 & 1 & 1 \\
\hline DBP & 9 & - & 2 (intron 1) \\
\hline ReverbA & 5 & 1 & 2 \\
\hline Dec-1 & 8 & 2 & 2 \\
\hline Decorin promoter & 8 & - & 4 \\
\hline Decorin intron & 6 & - & 4 \\
\hline
\end{tabular}

The last column of the table lists the number of GREs selected for analysis of activity by luciferase assay. in the presence of CLOCK or Bmal, but not both (Fig. 2C). Promoter regions for CRY2 ( -7920 to -6381$)$, Bmal ( -649 to 0 ) and PER2 ( -802 to 0 ) were significantly induced by aldosterone and this was largely preserved when Bmal or CLOCK were overexpressed separately. The exception being reduced CRY2 transactivation by CLOCK alone (Fig. 2D). Furthermore, co-expression of CLOCK plus Bmal inhibited MR-dependent transactivation of CRY2 and Per2; Bmal alone did not modify MR-dependent transactivation whereas CLOCK alone inhibited CRY2 (Fig. 2D and F). In contrast, Bmal transactivation in response to aldosterone was equivalent in the presence and absence of CLOCK and/or Bmal (Fig. 2E). Other promoter regions we investigated were unresponsive to aldosterone (data not shown).

Luciferase reporter constructs for the MR target genes GiLZ and decorin were evaluated for combined regulation by MR, CLOCK and/or Bmal. Transactivation of GiLZ by $10 \mathrm{nM}$ aldosterone was equivalent in the presence and absence of CLOCK and Bmal (Fig. 3). Two regions of decorin, -9423 to -8601 and 1967-3140 (intron) were tested; decorin -9423 to -8601 was not regulated by MR signalling (data not shown), whereas decorin 1967-3140 was regulated by aldosterone, but only when CLOCK, Bmal or both were overexpressed. These data suggest that subsets of MR target genes are regulated by a composite of MR and circadian transcription factors (Fig. 3).

\section{Over expression CLOCK and Bmal modifies MR-mediated transcription of endogenous genes}

Given that CLOCK and Bmal modify MR-dependent transactitvation of luciferase reporter genes, we evaluated the effect of overexpression of Bmal and CLOCK on aldosterone-mediated, MR-dependent gene expression in $\mathrm{H} 9 \mathrm{c} 2$ cells. A total of $10 \mathrm{nM}$ aldosterone significantly increased PER1 mRNA levels at $5 \mathrm{~h}$, whereas co-expression of CLOCK and Bmal reduced MR-mediated gene expression. The mRNA levels were increased for MR target gene SGK1 but they did not reach significance (Fig. 4A and B). Although aldosterone alone only modestly increased PER2 and decorin mRNA levels, in the presence of CLOCK overexpression aldosterone significantly reduced mRNA levels for both genes (Fig. 5C and D). Given that CLOCK alone induced PER2 and decorin, the response to aldosterone may reflect a change in the temporal pattern of gene expression. Taken together these data provide proof of concept that net gene expression in response to combined MR/circadian signalling is dependent upon the specific promoter. 

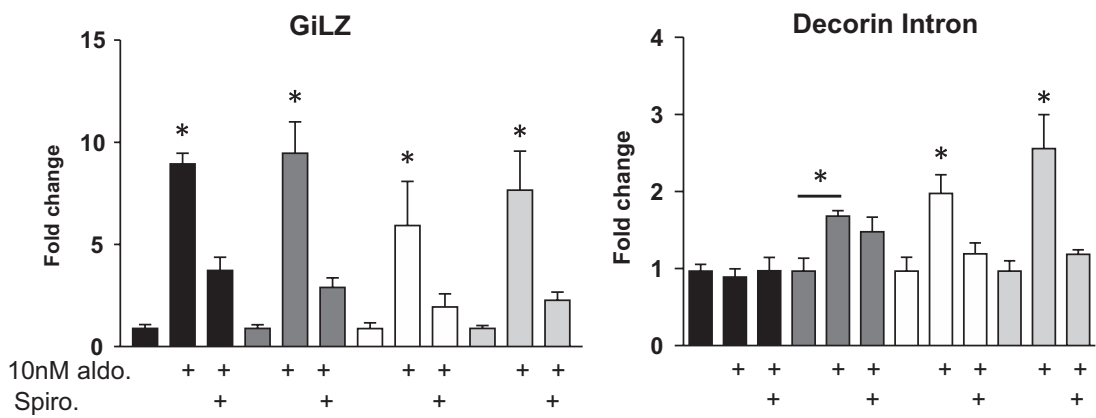

\section{Figure 3}

Regulation of MR target gene promoters decorin (DCN) and GiLZ by aldosterone is modified by overexpression of Bmal (dark grey bars), Clock (white bars) or Bmal + CLOCK (light grey bars) vs empty vector (black bars). CLOCK and Bmal repress basal transactivation of DCN and GiLZ promoter elements. Data are presented as mean \pm s.E.M. $n=3$ experiments performed in triplicate $* P<0.5$ vs vehicle.

\section{Aldosterone regulation of endogenous circadian genes in the heart is dependent on time of day}

These experiments had two goals: to determine if the MR regulates expression of cardiac circadian clock genes and to establish whether time of day, and thus endogenous circadian clock signalling is associated with changes in MR-mediated gene expression. Cardiomyocyte MRs are not selective and bind glucocorticoids; aldosterone was therefore used here as a 'pure' MR agonist to identify MR-selective gene regulation of circadian and MR target genes in intact C57bl6 mice. The dose used was based on previous acute in vivo gene expression studies and administered to adrenal intact animals, a key difference with previous acute receptor regulation studies (Tanaka et al. 2007). This approach was necessary given that adrenalectomy would adversely affect the circadian clock and confound the study. In our vehicle-treated mice, the MR and GR would thus be activated by endogenous corticosterone alone whereas in the aldosterone-treated mice, cortisol would bind GR while aldosterone was at sufficient levels to bind to the MR.

Aldosterone was administered $12 \mathrm{~h}$ apart $(08: 00 \mathrm{~h}$ or 20:00 h) when circadian clock signalling shows maximal difference in terms of CLOCK and Bmal activity (Durgan et al. 2005) and with gene expression determined before and $4 \mathrm{~h}$ after aldosterone or vehicle treatment. The expression of all circadian genes showed regulation consistent with their expected circadian pattern of expression: that is, $08: 00-20: 00 \mathrm{~h}, 08: 00-12: 00 \mathrm{~h}$, 20:00-00:00 h and so on (Fig. 5A, B, C, D, E, F, G and Supplementary Fig. 5). When administered at 08:00 h, bolus aldosterone significantly regulated PER1, PER2 and TEF mRNA levels $4 \mathrm{~h}$ later when compared to vehicle. Aldosterone administered at $20: 00 \mathrm{~h}$ did not regulate PER1 and PER2 (Fig. 5 and Supplementary Fig. 4). In contrast NPAS, Timeless and MR target genes GiLZ, SGK1 and decorin were not significantly regulated by time or ligand; we also detected time-dependent changes in the expression of MR (Supplementary Figs 4 and 5).
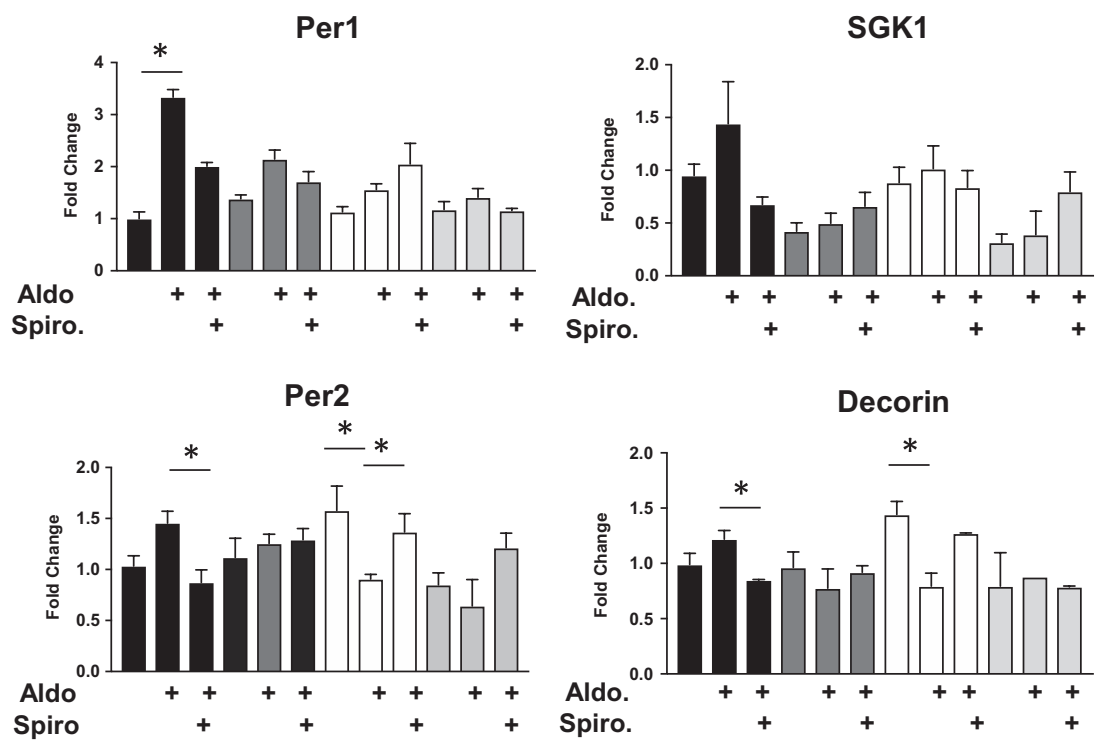

\section{Figure 4}

Overexpression of Bmal and CLOCK modifies endogenous MR target gene expression after $5 \mathrm{~h}$ in $\mathrm{H} 9 \mathrm{c} 2$ cells. Bmal (dark grey bars), Clock (white bars) or Bmal + CLOCK (light grey bars) vs empty vector (black bars). Data presented are mean of three independent experiments and are presented as mean \pm S.E.M. $* P<0.5$ vs vehicle. 
A

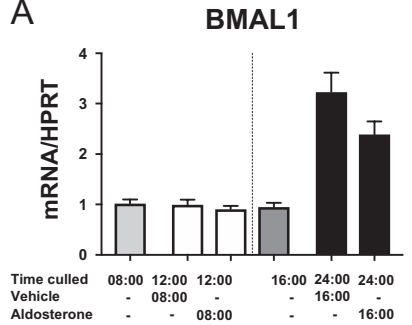

E

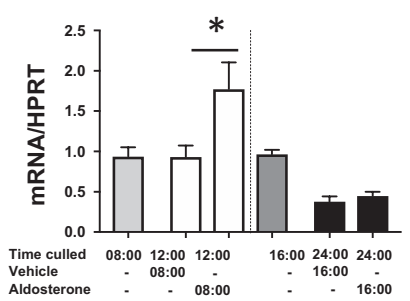

B

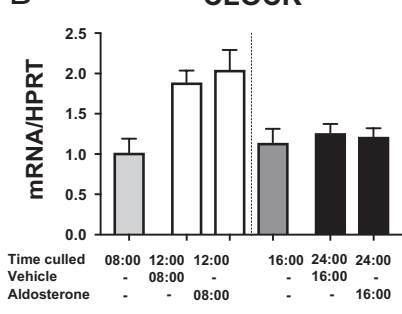

$\mathrm{F}$

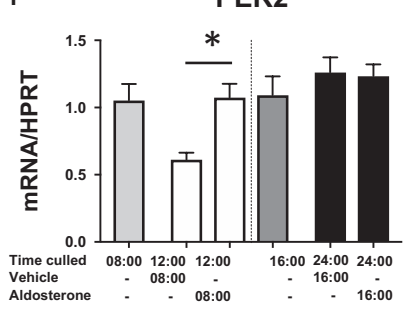

C
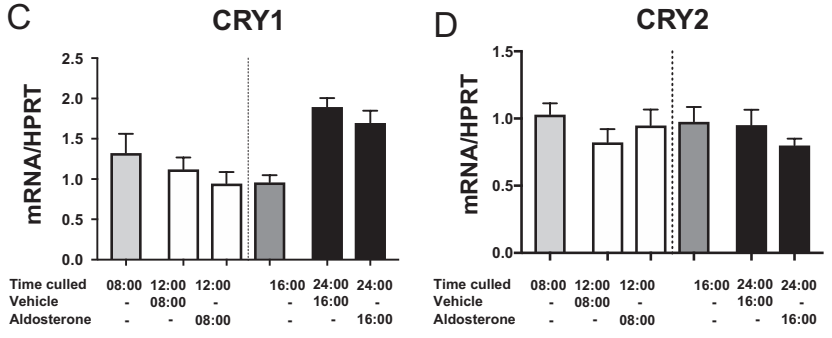

G

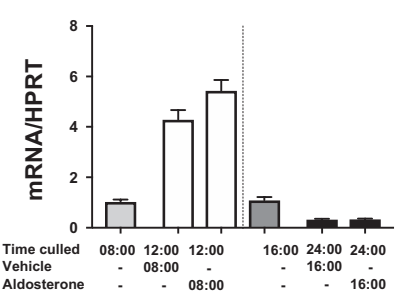

Figure 5

Circadian gene expression in vehicle-treated animals was regulated over the $4 \mathrm{~h}$ between 08:00 $\mathrm{h}$ and 12:00 $\mathrm{h}$ or 20:00 $\mathrm{h}$ and 00:00 $\mathrm{h}$. Circadian genes showed the expected regulation over time. A bolus dose of aldosterone given at 08:00 h significantly upregulated PER1 (E) and PER2 (F) at $4 \mathrm{~h}$ vs vehicle. Aldosterone administered at 20:00 h was without effect. Mean \pm S.E.M.; data are representative of two independent RT and PCR experiments where $n=8-10 /$ group, $* P<0.01$ vs $08: 00 \mathrm{~h}$ and vehicle at $12: 00 \mathrm{~h}$.

\section{Endogenous circadian genes in the liver are strongly regulated by time of day, but not by aldosterone}

Expression of circadian genes and MR target genes were also investigated in liver samples given that the liver shows strong circadian expression of the molecular clock and that hepatocytes do not express the MR. The liver showed strong endogenous circadian patterns of gene expression from $08: 00 \mathrm{~h}$ to $20: 00 \mathrm{~h}$, from $08: 00 \mathrm{~h}$ to $12: 00 \mathrm{~h}$ or from 20:00h to 00:00h (Supplementary Figs 6 and 7). Treatment with aldosterone did not change the expression of any of these genes consistent with the absence of MR in hepatocytes. The MR target genes GiLZ and decorin also exhibited a circadian pattern of expression between 08:00 h and 12:00h (Supplementary Fig. 8).

\section{Discussion}

Our data show for the first time that aldosterone-mediated MR signalling in vivo can have differential effects on cardiac gene expression when administered at dawn (08:00h) vs dusk (20:00h) highlighting the potential for circadian variation in the transcriptional machinery to influence MR transcriptional responses in the heart. For in vivo experiments presented here aldosterone was used as an experimental tool to determine responses that were unique to the MR. Cardiac MR are predominantly occupied by cortisol, even at the nadir of cortisol release or in some cases in the presence of an aldosterone secreting tumour. The present study also provides novel insights into MR-dependent regulation of the cardiac tissue clock; MR activation in cardiomyocytes regulates the pattern of expression of select circadian genes (CRY1, PER1, PER2 and ReverbA) over $24 \mathrm{~h}$ in a ligand-dependent manner. Moreover, our data show combined MR and CLOCK/Bmal transcriptional responses that are promoter and ligand specific, demonstrating that MR transcriptional responses are significantly modified by circadian signalling at some promoters. Additional studies are needed to characterise the cellular context in cardiac, and potentially other, cells types that may favour combined MR and CLOCK/ Bmal regulation of these targets. Cardiovascular disease is frequently associated with disruption of the intrinsic circadian machinery within cardiomyocytes by diseasespecific dysregulation of pathways or via SCN-directed, HPA axis-driven misalignment of peripheral circadian timing (e.g. as in shift work) (Durgan et al. 2005, Durgan \& Young 2010). Inappropriate or pathogenic MR signalling programmes may compound disordered circadian transcription factor signalling to promote tissue injury and remodelling. Taken together our data provide evidence for reciprocal regulation of MR and the circadian clock in cardiomyocytes. These data may have important implications for optimization of current therapeutics targeting the MR, and also for novel target identification for the treatment of cardiovascular diseases. 


\section{MR signalling modifies $24 \mathrm{~h}$ patterns of circadian gene expression}

Our data show that for cardiomyocytes, in which the autonomous circadian clock has been synchronised, the addition of aldosterone or corticosterone modifies the $24 \mathrm{~h}$ pattern of expression of endogenous circadian genes - CRY1, PER1/2 and ReverbA. Transcriptional regulation of PER1 and PER2 by both aldosterone and the glucocorticoids is established (Tanaka et al. 2007, Richards et al. 2013, Le Billan et al. 2015). Active GRE sequences in the promoter regions of both genes have been identified (Reddy et al. 2012, Han et al. 2014) and ChIPseq approaches have characterised the MR-dependent regulation of PER1 (Gumz et al. 2009). Using in silico analyses and luciferase reporter assays, we identified additional GREs in the promoters of multiple circadian genes and further demonstrated that Bmal and CLOCK can significantly regulate ligand-mediated, MR-directed transcription of these targets. Moreover, we have shown that responses differ for aldosterone and cortisol. These data are more compelling when considered in conjunction with the in vivo demonstration that time of day significantly modifies aldosterone-dependent regulation of PER1 and PER2.

Corticosterone treatment $(10 \mathrm{nM})$ in a synchronised cardiomyocyte cell line produced a 'phase advance' in the $24 \mathrm{~h}$ expression pattern of CRY1, PER1 and PER2, whereas the PER2 response to aldosterone was apparent at $12-18 \mathrm{~h}$ and indicated an overall 'phase delay'. In contrast both ligands induced a similar phase delay in ReverbA expression in synchronised $\mathrm{H} 9 \mathrm{c} 2$ cells. These data not only provide evidence that ReverbA is an MR target but also demonstrate that the MR, as well as the GR, can transduce corticosterone signalling for select circadian genes (Caratti et al. 2018). Taken together these data have important implications for physiological vs pathological regulation of circadian gene targets where fluctuations between ligand availability, receptor levels and thus MR and GR homodimers or MR/GR heterodimers contribute to the net regulatory outcome (Le Billan et al. 2015). These data are consistent with circadian regulation of MR and GR in neurons where it is recognised that MR is fully occupied by ligand over $24 \mathrm{~h}$ but ligand occupancy of the GR fluctuates (Joels \& de Kloet 2017). Although MR blockade is sufficient to reverse ligand-mediated changes in cardiac circadian gene expression, it is important to note that MR blockade cannot modify central circadian timing due to the absence of the MR (and GR) in the SCN. An impact on circadian timing can occur via modulation of HPA responsiveness, for example in the hypothalamus (Joels \& de Kloet 2017).

\section{Direct transcriptional regulation of circadian genes is the composite effect of MR and circadian transcription factor signalling}

In silico analyses of both known and putative GRE and E-box sequences established potential regions in the $10 \mathrm{~kb}$ promoter regions of circadian clock genes for combined transcriptional responses to MR and circadian transcription factors. Although a putative MRE has been reported (Le Billan et al. 2015), we did not detect this sequence in any promoter sequence. Our novel reporter assays containing selected regions of the circadian promoters revealed several patterns of regulation in response to combined MR and circadian transcription factors CLOCK and Bmal. First, we established MR transactivation of Bmal, CRY2 and PER2 and modest transrepression of the proximal CLOCK promoter region. These assays are performed in non-synchronised cells, incubated with serum-free media. The MR responses to aldosterone activation will not be affected by circadian clock phase in this cell population because cells will be distributed throughout the entire circadian cycle. However, the acute effects of the circadian transcription factors CLOCK and Bmal1 on MR transcriptional responses showed multiple patterns of response: inhibition (proximal CLOCK, CRY2), induction (distal CLOCK, CRY1) and no change (Bmal, PER2). These data represent net luciferase responses at $16 \mathrm{~h}$ but highlight the potential for promoter-specific transcriptional outcomes for ligand-mediated transactivation when combined with other key transcriptional regulators.

A key observation is that not all circadian genes are regulated by ligand-activated MR. There are several mechanisms that may explain this observation. First, different promoter regions will contain different GRE sequences and MR regulate transcription via a subset of GREs (Le Billan et al. 2015). Secondly, in cells where MR is predominantly bound by cortisol (or corticosterone in rodents), DNA binding at GREs will potentially be by different contributions of MR/MR, MR/GR or GR/GR dimers due to variable MR expression over $24 \mathrm{~h}$. Variable ligand binding of the GR from the nadir of cortisol secretion where it is virtually unoccupied to high occupancy at the transition of rest to active will also make a significant contribution to the ratio of MR vs GR. Thirdly, coregulatory proteins for the MR can be ligand dependent (Yang et al. 2011), further modifying transcriptional responses; coregulator protein availability may also fluctuate over the day.

An interaction between MR signalling and circadian has been reported (Tanaka et al. 2007). Moreover, So et al. 
previously identified seven GREs in the PER1 promoter, of which only two sites were determined to be continuously occupied by the GR; the other GREs may also respond to GR and MR depending on ligand and receptor availability (Reddy et al. 2009, So et al. 2009). The novel GREs identified in the present were responsive to MR transactivation; whether there is a role for MR/GR dimers at one or more of these sites across the family of circadian genes remains to be formally demonstrated (Mahfouz et al. 2016). Together with our previous data for the PER1 promoter, which shows an additive response to MR plus CLOCK/Bmal signalling (Fletcher et al. 2017), our data highlight the potential for the autonomous circadian clock to selectively regulate MR transcriptional responses in vivo, adding a level of complexity offering potential mechanism for MR signalling events across the day.

\section{Combined transcriptional regulation of MR target genes by CLOCK, Bmal and the MR}

It is estimated that up to $40-60 \%$ of the genome is regulated in a circadian manner (Young 2009, Bellet \& Sassone-Corsi 2010). Given that the circadian release of glucocorticoids is a primary neurohormonal entrainment signal for peripheral tissue clocks, it follows that many GR target genes will show ligand-dependent circadian variation in their expression. Online databases for wholegenome circadian expression of genes can provide a guide to tissue-specific regulation of individual MR target genes under physiological or specific pathological conditions. However, the impact of circadian signalling per se on ligand-mediated MR target genes, other than for PER1 and PER2, has not been formally investigated.

A number of MR-regulated genes are known targets of the molecular clock: PAI-1, sgk1 and GiLZ (Schoenhard et al. 2003, Gumz et al. 2009, Oishi et al. 2009). MR target gene GiLZ and novel MR target gene decorin contained both GRE/E-box regulatory elements, although only MR regulation of decorin was regulated by CLOCK/Bmal. These data provide proof of principle that some MR target genes have the capacity for combined MR and circadian signalling and that for other genes, perhaps time-of-day signalling factors are superseded by short-term responses to volume depletion or stress.

CLOCK and Bmal have many key functions beyond their role as core circadian signalling partners. They thus have the potential to regulate MR target promoters via mechanisms other than acting as a heterodimer to modify the net MR transcriptional response. CLOCK and Bmal independently regulate target genes through direct promoter interactions and in the case of CLOCK, via histone acetyltransferase (HAT) activity, which may independently impact MR-dependent signalling as has been demonstrated for the GR (Curtis et al. 2004, Doi et al. 2006). For example, CLOCK-mediated acetylation of multiple lysine residues in the hinge region of the GR prevents nuclear translocation and disrupts normal GR/GRE interactions, the net effect being a significant change to GR transrepression of NF-кB and the transcriptional control of anti-inflammatory actions (Nader et al. 2009).

\section{Circadian regulation of the MR in cardiomyocytes}

We also present in vivo data demonstrating differential MR-dependent transcription of circadian genes in the heart between the nominal 'dawn and dusk' time points (lights on vs lights off). These novel data highlight the potential for circadian variation in transcription factor availability, cell function and other factors to have a significant impact on the outcome of MR signalling. For other nuclear receptors at least, circadian variation in receptor function has important implications for timing of therapeutic administration. For example, it is well known that administration of therapeutic doses of glucocorticoids at specific times in the circadian cycle increases the efficacy of and limits unwanted side effects due to differential effects of CLOCK on GR transrepression/transcriptional effects. Our data suggest that MR antagonist use may be refined by observing circadian differences in activation.

CLOCK:Bmal1 regulation of the GR is established at the transcriptional and protein level; elevated CLOCK or CRY1/2 suppress GR levels by direct protein-protein interactions (Nader et al. 2009, Lamia et al. 2011, Gibbs et al. 2014, Goriki et al. 2014, Han et al. 2014). More recently Caratti et al. (2018) demonstrated that efficient and time-of-day-dependent GR recruitment to chromatin required ReverbA maintaining histone acetylation.

Receptor availability may also play a key role in the circadian variation of aldosterone-mediated gene expression in the heart, although cardiac mRNA levels for the MR at the time of bolus aldosterone treatment (either 08:00 h or 20:00 h) were similar. Whole-genome analysis of circadian transcriptional responses in 13 human tissues, including atrial tissue, found that the MR is a ubiquitous cycling gene (Ruben et al. 2018); however to the best of our knowledge CLOCK:Bmal1 regulation of the MR has not been demonstrated. While the present data demonstrate the net effect of two sets of transcription factors acting via their cognate response elements that have equally important outcomes in terms of target gene 
regulation, other mechanisms for circadian modification of MR transcription, that is protein-protein interaction, are also plausible. Of note, the lysine residues in the hinge region of GR targeted by CLOCK are conserved in the MR, and $\mathrm{PR}$ and $\mathrm{AR}$, raising the possibility that reduced MR transcription for some promoters is mediated by similar CLOCK-dependent regulatory processes (Kino \& Chrousos 2011b).

\section{GR vs MR signalling: ligand vs receptor}

Basal corticosteroid release follows a circadian and ultradian rhythm via neurohormonal signals from SCN regulation of the HPA axis. In addition to the circadian release of cortisol, pulsatile release of corticosteroids ensures optimal responsiveness of HPA axis reactivity to environmental challenges. Aldosterone levels also show a circadian pattern of release, in part due to modest regulation by ACTH, and by posture and sodium/water intake. For the MR in particular antagonist-binding potentiates the HPA axis in acute experiments (Cornelisse et al. 2011). All of these events contribute to the availability of corticosteroid and thus the ratio of MR/GR activation, in non-epithelial tissues. While there are potential differences between the brain and peripheral tissues, such as the heart, given differences in ligand access across the blood-brain barrier, there are similarities between the heart and neural MR/GR signalling in terms of the ability of the MR to bind both ligands with high affinity and without the presence of prereceptor metabolism of cortisol. A similar balance between MR and GR signalling in response to fluctuating cortisol levels may thus operate in the heart to modify circadian signalling outcomes. While glucocorticoids acting via the GR are regarded as a 'zeitgeber' or 'timekeeper' of the circadian clock, equivalent studies for the MR are limited to PER1 and PER2 regulation. We have shown in the present study that the MR regulates core circadian genes in response to high levels of aldosterone to compete with endogenous glucocorticoids in vivo, and to aldosterone and corticosterone in vitro, and that circadian transcription factors in turn modify the MR transcriptional response in the heart. This suggests an integrated and complex reciprocal regulatory of MR signalling in the heart, which will include cortisol/corticosterone actions given that cardiac MR are non-selective for aldosterone. As noted, cardiac MR, and other non-epithelial MR, are predominantly if not exclusively occupied by circulating cortisol/corticosterone regardless of circadian variation in serum levels and binding to corticosterone-binding globulin (CBG) (Joels \& de Kloet 2017). In contrast, binding and activation of the GR is directly regulated by circadian variation in cortisol/corticosterone levels. Given that the MR is continuously occupied, circadian regulation of MR levels allow for variable MR responses (Joels \& De Kloet 1992). Our data for variable MR levels in the heart over $24 \mathrm{~h}$ are consistent with a recent study in humans and mice that showed MR (NR3C2) have a circadian pattern of expression (Ruben et al. 2018).

Thus MR availability, ligand availability and the formation of heterodimers with the GR vs homodimers all contribute to fine-tuning regulation of circadian and other genes in time-of-day-dependent manner. It is established that treating patients with glucocorticoids should consider the endogenous oscillatory profile of corticosteroids to achieve physiological regulation and avoid unwanted side effects. There may now be an argument to incorporate normal circadian MR regulation in the administration of MR antagonist therapies. The present data thus offer novel signalling mechanisms for inappropriate MR activation leading to dysregulated signalling of the cardiac tissue circadian clock, which in turn impacts cardiac inflammation, fibrosis, hypertrophy and the response to ischaemia.

\section{Supplementary data}

This is linked to the online version of the paper at https://doi.org/10.1530/ JOE-18-0584.

\section{Declaration of interest}

$M J Y$ and $P J F$ have received independent research funding from AstraZeneca for work that is unrelated to the current study. The other authors have nothing to disclose.

\section{Funding}

This work was supported by a grants-in-aid from the National Health and Medical Research Council of Australia (GNT1001034). The Hudson Institute is supported by the Victorian Government's Operational Infrastructure Support (OIS) Program.

\section{References}

Balsalobre A, Damiola F \& Schibler U 1998 A serum shock induces circadian gene expression in mammalian tissue culture cells. Cell 93 929-937. (https://doi.org/10.1016/S0092-8674(00)81199-X)

Bellet MM \& Sassone-Corsi P 2010 Mammalian circadian clock and metabolism - the epigenetic link. Journal of Cell Science $\mathbf{1 2 3}$ 3837-3848. (https://doi.org/10.1242/jcs.051649)

Bienvenu LA, Reichelt ME, Morgan J, Fletcher EK, Bell JR, Rickard AJ, Delbridge LM \& Young MJ 2015 Cardiomyocyte mineralocorticoid 
receptor activation impairs acute cardiac functional recovery after ischemic insult. Hypertension 66 970-977. (https://doi.org/10.1161/ HYPERTENSIONAHA.115.05981)

Caratti G, Iqbal M, Hunter L, Kim D, Wang P, Vonslow RM, Begley N, Tetley AJ, Woodburn JL, Pariollaud M, et al. 2018 REVERBa couples the circadian clock to hepatic glucocorticoid action. Journal of Clinical Investigation 128 4454-4471. (https://doi.org/10.1172/JCI96138)

Chandler VL, Maler BA \& Yamamoto KR 1983 DNA sequences bound specifically by glucocorticoid receptor in vitro render a heterologous promoter hormone responsive in vivo. Cell 33 489-499. (https://doi. org/10.1016/0092-8674(83)90430-0)

Cheon S, Park N, Cho S \& Kim K 2013 Glucocorticoid-mediated Period2 induction delays the phase of circadian rhythm. Nucleic Acids Research 41 6161-6174. (https://doi.org/10.1093/nar/gkt307)

Cornelisse S, Joels M \& Smeets T 2011 A randomized trial on mineralocorticoid receptor blockade in men: effects on stress responses, selective attention, and memory. Neuropsychopharmacology 36 2720-2728. (https://doi.org/10.1038/npp.2011.162)

Curtis AM, Seo SB, Westgate EJ, Rudic RD, Smyth EM, Chakravarti D, FitzGerald GA \& McNamara P 2004 Histone acetyltransferasedependent chromatin remodeling and the vascular clock. Journal of Biological Chemistry 279 7091-7097. (https://doi.org/10.1074/jbc. M311973200)

Doi M, Hirayama J \& Sassone-Corsi P 2006 Circadian regulator CLOCK is a histone acetyltransferase. Cell 125 497-508. (https://doi. org/10.1016/j.cell.2006.03.033)

Durgan DJ \& Young ME 2010 The cardiomyocyte circadian clock: emerging roles in health and disease. Circulation Research 106 647-658. (https://doi.org/10.1161/CIRCRESAHA.109.209957)

Durgan DJ, Hotze MA, Tomlin TM, Egbejimi O, Graveleau C, Abel ED, Shaw CA, Bray MS, Hardin PE \& Young ME 2005 The intrinsic circadian clock within the cardiomyocyte. American Journal of Physiology: Heart and Circulatory Physiology 289 H1530-H1541. (https://doi.org/10.1152/ajpheart.00406.2005)

Fletcher EK, Morgan J, Kennaway DR, Bienvenu LA, Rickard AJ, Delbridge LMD, Fuller PJ, Clyne CD \& Young MJ 2017 Deoxycorticosterone/salt-mediated cardiac inflammation and fibrosis are dependent on functional CLOCK signaling in male mice. Endocrinology 158 2906-2917. (https://doi.org/10.1210/en.2016-1911)

Fuller PJ \& Young MJ 2005 Mechanisms of mineralocorticoid action. Hypertension 46 1227-1235. (https://doi.org/10.1161/01. HYP.0000193502.77417.17

Gibbs J, Ince L, Matthews L, Mei J, Bell T, Yang N, Saer B, Begley N, Poolman T, Pariollaud M, et al. 2014 An epithelial circadian clock controls pulmonary inflammation and glucocorticoid action. Nature Medicine 20 919-926. (https://doi.org/10.1038/nm.3599)

Goriki A, Hatanaka F, Myung J, Kim JK, Yoritaka T, Tanoue S, Abe T, Kiyonari H, Fujimoto K, Kato Y, et al. 2014 A novel protein, CHRONO, functions as a core component of the mammalian circadian clock. PLoS Biology 12 e1001839. (https://doi.org/10.1371/ journal.pbio.1001839)

Gumz ML, Stow LR, Lynch IJ, Greenlee MM, Rudin A, Cain BD, Weaver DR \& Wingo CS 2009 The circadian clock protein period 1 regulates expression of the renal epithelial sodium channel in mice. Journal of Clinical Investigation 119 2423-2434. (https://doi. org/10.1172/JCI36908)

Han DH, Lee YJ, Kim K, Kim CJ \& Cho S 2014 Modulation of glucocorticoid receptor induction properties by core circadian clock proteins. Molecular and Cellular Endocrinology 383 170-180. (https:// doi.org/10.1016/j.mce.2013.12.013)

Joels M \& De Kloet ER 1992 Coordinative mineralocorticoid and glucocorticoid receptor-mediated control of responses to serotonin in rat hippocampus. Neuroendocrinology 55 344-350. (https://doi. org/10.1159/000126135)

Joels M \& de Kloet ER 201730 YEARS OF THE MINERALOCORTICOID RECEPTOR: The brain mineralocorticoid receptor: a saga in three episodes. Journal of Endocrinology 234 T49-T66. (https://doi org/10.1530/JOE-16-0660)

Kino T \& Chrousos GP 2011 a Circadian CLOCK-mediated regulation of target-tissue sensitivity to glucocorticoids: implications for cardiometabolic diseases. Endocrine Development 20 116-126. (https:// doi.org/10.1159/000321232)

Kino T \& Chrousos GP $2011 b$ Acetylation-mediated epigenetic regulation of glucocorticoid receptor activity: circadian rhythm-associated alterations of glucocorticoid actions in target tissues. Molecular and Cellular Endocrinology 336 23-30. (https://doi.org/10.1016/j. mce.2010.12.001)

Lamarre-Cliche M, de Champlain J, Lacourciere Y, Poirier L, Karas M \& Larochelle P 2005 Effects of circadian rhythms, posture, and medication on renin-aldosterone interrelations in essential hypertensives. American Journal of Hypertension 18 56-64. (https://doi. org/10.1016/j.amjhyper.2004.08.025)

Lamia KA, Papp SJ, Yu RT, Barish GD, Uhlenhaut NH, Jonker JW, Downes M \& Evans RM 2011 Cryptochromes mediate rhythmic repression of the glucocorticoid receptor. Nature $\mathbf{4 8 0} 552-556$. (https://doi.org/10.1038/nature10700)

Le Billan F, Khan JA, Lamribet K, Viengchareun S, Bouligand J, Fagart J \& Lombes M 2015 Cistrome of the aldosterone-activated mineralocorticoid receptor in human renal cells. FASEB Journal 29 3977-3989. (https://doi.org/10.1096/fj.15-274266)

Mahfouz A, Lelieveldt BP, Grefhorst A, van Weert LT, Mol IM, Sips HC van den Heuvel JK, Datson NA, Visser JA, Reinders MJ, et al. 2016 Genome-wide coexpression of steroid receptors in the mouse brain identifying signaling pathways and functionally coordinated regions PNAS 113 2738-2743. (https://doi.org/10.1073/pnas.1520376113)

Nader N, Chrousos GP \& Kino T 2009 Circadian rhythm transcription factor CLOCK regulates the transcriptional activity of the glucocorticoid receptor by acetylating its hinge region lysine cluster: potential physiological implications. FASEB Journal 23 1572-1583. (https://doi.org/10.1096/fj.08-117697)

Oishi K, Miyazaki K, Uchida D, Ohkura N, Wakabayashi M, Doi R, Matsuda J \& Ishida N 2009 PERIOD2 is a circadian negative regulator of PAI-1 gene expression in mice. Journal of Molecular and Cellular Cardiology 46 545-552. (https://doi.org/10.1016/j.yjmcc.2009.01.001)

Reddy AB, Maywood ES, Karp NA, King VM, Inoue Y, Gonzalez FJ, Lilley KS, Kyriacou CP \& Hastings MH 2007 Glucocorticoid signaling synchronizes the liver circadian transcriptome. Hepatology $\mathbf{4 5}$ 1478-1488. (https://doi.org/10.1002/hep.21571)

Reddy TE, Pauli F, Sprouse RO, Neff NF, Newberry KM, Garabedian MJ \& Myers RM 2009 Genomic determination of the glucocorticoid response reveals unexpected mechanisms of gene regulation. Genome Research 19 2163-2171. (https://doi.org/10.1101/gr.097022.109)

Reddy TE, Gertz J, Crawford GE, Garabedian MJ \& Myers RM 2012 The hypersensitive glucocorticoid response specifically regulates period 1 and expression of circadian genes. Molecular and Cellular Biology 32 3756-3767. (https://doi.org/10.1128/MCB.00062-12)

Richards J, Cheng KY, All S, Skopis G, Jeffers L, Lynch IJ, Wingo CS \& Gumz ML 2013 A role for the circadian clock protein Per1 in the regulation of aldosterone levels and renal $\mathrm{Na}+$ retention. American Journal of Physiology: Renal Physiology 305 F1697-F1704. (https://doi. org/10.1152/ajprenal.00472.2013)

Rickard AJ, Morgan J, Bienvenu LA, Fletcher EK, Cranston GA, Shen JZ, Reichelt ME, Delbridge LM \& Young MJ 2012 Cardiomyocyte mineralocorticoid receptors are essential for deoxycorticosterone/saltmediated inflammation and cardiac fibrosis. Hypertension $\mathbf{6 0}$ 1443-1450. (https://doi.org/10.1161/ HYPERTENSIONAHA.112.203158)

Ruben MD, Wu G, Smith DF, Schmidt RE, Francey LJ, Lee YY, Anafi RC \& Hogenesch JB 2018 A database of tissue-specific rhythmically expressed human genes has potential applications in circadian medicine. Science Translational Medicine 10 eaat8806. (https://doi. org/10.1126/scitranslmed.aat8806 https://joe.bioscientifica.com

https://doi.org/10.1530/JOE-18-0584 (c) 2019 Society for Endocrinology Published by Bioscientifica Ltd. Printed in Great Britain 
Schoenhard JA, Smith LH, Painter CA, Eren M, Johnson CH \& Vaughan DE 2003 Regulation of the PAI-1 promoter by circadian clock components: differential activation by BMAL1 and BMAL2. Journal of Molecular and Cellular Cardiology 35 473-481. (https://doi. org/10.1016/S0022-2828(03)00051-8)

Singletary JH, Chan D, Samani NJ \& Chong NW 2008 The canonical E-box motif: a target for glucocorticoid action that drives rhythmic mouse Pai-1 transcription in vitro. Gene $\mathbf{4 2 0} 42-47$. (https://doi. org/10.1016/j.gene.2008.05.004)

So AY, Bernal TU, Pillsbury ML, Yamamoto KR \& Feldman BJ 2009 Glucocorticoid regulation of the circadian clock modulates glucose homeostasis. PNAS 106 17582-17587. (https://doi.org/10.1073/ pnas.0909733106)

Stow LR, Richards J, Cheng KY, Lynch IJ, Jeffers LA, Greenlee MM, Cain BD, Wingo CS \& Gumz ML 2012 The circadian protein period 1 contributes to blood pressure control and coordinately regulates renal sodium transport genes. Hypertension 59 1151-1156. (https://doi. org/10.1161/HYPERTENSIONAHA.112.190892)

Takahashi JS \& Zatz M 1982 Regulation of circadian rhythmicity. Science 217 1104-1111. (https://doi.org/10.1126/science.6287576)

Tanaka K, Ashizawa N, Kawano H, Sato O, Seto S, Nishihara E, Terazono H, Isomoto S, Shinohara K \& Yano K 2007 Aldosterone induces circadian gene expression of clock genes in H9c2 cardiomyoblasts. Heart and Vessels 22 254-260. (https://doi org/10.1007/s00380-006-0968-3)

Yamamoto Y, Yagita K \& Okamura H 2005 Role of cyclic mPer2 expression in the mammalian cellular clock. Molecular and Cellular Biology 25 1912-1921. (https://doi.org/10.1128/МСB.25.5.19121921.2005)

Yang J, Chang CY, Safi R, Morgan J, McDonnell DP, Fuller PJ, Clyne CD \& Young MJ 2011 Identification of ligand-selective peptide antagonists of the mineralocorticoid receptor using phage display. Molecular Endocrinology 25 32-43. (https://doi.org/10.1210/me. 2010-0193)

Yoshitane H, Takao T, Satomi Y, Du NH, Okano T \& Fukada Y 2009 Roles of CLOCK phosphorylation in suppression of E-box-dependent transcription. Molecular and Cellular Biology 29 3675-3686. (https:// doi.org/10.1128/MCB.01864-08)

Young ME 2009 Anticipating anticipation: pursuing identification of cardiomyocyte circadian clock function. Journal of Applied Physiology 107 1339-1347. (https://doi.org/10.1152/japplphysiol.00473.2009)

Ziera T, Irlbacher H, Fromm A, Latouche C, Krug SM, Fromm M, Jaisser F \& Borden SA 2009 Cnksr3 is a direct mineralocorticoid receptor target gene and plays a key role in the regulation of the epithelial sodium channel. FASEB Journal 23 3936-3946. (https://doi. org/10.1096/fj.09-134759)

Received in final form 23 January 2019

Accepted 28 January 2019

Accepted Preprint published online 28 January 2019 (c) 2019 Society for Endocrinology Published by Bioscientifica Ltd.
Printed in Great Britain 